\title{
Measurement Based Spatial Characteristics of MPCs in Train-to-Train Propagation
}

\author{
Paul Unterhuber*, Thomas Jost*, Wei Wang*, Thomas Kürner ${ }^{\dagger}$ \\ *Institute of Communications and Navigation, German Aerospace Center (DLR), Oberpfaffenhofen, 82234 Wessling, \\ Germany, paul.unterhuber@dlr.de \\ $\dagger$ Institute for Communications Technology, Technische Universität Braunschweig, 38106 Braunschweig, Germany
}

\begin{abstract}
Railway operators for high speed trains, commuter trains or subways request for technologies to increase the density and efficiency on their rails. One of those future technologies is virtual coupling based on train-to-train (T2T) wireless communication links. For the design of a wireless communication system, the knowledge of wave propagation is crucial. To characterize the propagation effects between two moving trains a T2T channel sounding measurement campaign was performed. To post-process the data, we use the Kalman enhanced super resolution tracking (KEST) algorithm to detect and track multipath components (MPCs). Using the single-input single-output measurement data recorded while the transmitter and receiver were in motion, the complex amplitude, the delay and the life time of individual MPCs can be estimated. First results of the post-processing step, the geometrical representation of MPCs using point scatterers and possibilities to identify scattering positions are presented in this paper.
\end{abstract}

Index Terms - train-to-train, high speed train, propagation, measurement, KEST, path tracking.

\section{INTRODUCTION}

Railway operators for high speed trains (HSTs), commuter trains or subways request for new technologies to increase the density and efficiency on their rails. One of those future technologies is virtual coupling. Nowadays, trains are connected with a mechanical coupler and electrical hard wired connectors. In comparison, virtual coupled trains will be connected only via a wireless communication link. Consequently, virtual coupling requires an ultra reliable low latency communication (URLLC) system between two or more trains. Low latency requirements can be fulfilled with direct links such as trainto-train (T2T), similar to car-to-car (C2C) communication. For design and testing, channel models are essential but hardly investigated for T2T. An overview of measurements and channel models is given in [1]. To characterize the propagation effects between two moving trains for frequencies above $1 \mathrm{GHz}$ and large bandwidths, a T2T channel sounding measurement campaign was performed. A unique data set for different environments, maneuvers and speeds was collected.

In case of multiple-input multiple-output (MIMO) based channel sounding measurements as performed in [2], the complex amplitude, delay, angle of departure (AoD) and angle of arrival (AoA) can be estimated. Hence, a geometrical representation of multipath components (MPCs) by scatterers in a $3 \mathrm{D}$ space can be achieved.
For single-input single-output (SISO) measurements, with a fixed transmitter (Tx) and moving receiver $(\mathrm{Rx})$ a virtual array approach can be applied as described in [3]. Hence, the complex amplitude, delay and the AoA can be estimated.

For SISO measurements, with moving Tx and moving Rx as described in [4], the virtual array approach is not feasible. Therefore, neither AoD nor AoA information can be extracted. In this context we provide first results on the spectral characteristics of MPCs and explain how this information might be used to locate objects influencing wave propagation. The Kalman enhanced super resolution tracking (KEST) algorithm is applied to detect and track individual MPCs.

The paper is structured as follows: In Section II we present the parameter estimation done by KEST and in Section III possible geometrical relations between consecutive snapshots. The T2T measurement campaign is briefly introduced in Section IV. We provide first results in Section V and conclude the paper in Section VI.

\section{PARAMETER ESTIMATION}

The measured channel impulse response (CIR) $h(t, \tau)$ at the time instant $t$ and delay $\tau$ can be modeled as a sum of Dirac impulses

$$
h(t, \tau)=\sum_{i=0}^{N} \alpha_{i}(t) \cdot \delta_{i}\left(\tau-\tau_{i}(t)\right),
$$

where $\alpha_{i}$ stands for the complex amplitude and $\tau_{i}$ for the delay of the $i$ th path [5].

KEST is a super resolution algorithm to estimate and track the channel parameters as introduced in [6]. The estimated parameters are the complex amplitude $\alpha_{i}(t)$ and the delay $\tau_{i}(t)$ of each path $i$ for each snapshot at time $t$. The tracking of individual paths provides the behavior of MPCs over time. In other words, the life time and the fading of paths can be analyzed. The KEST algorithm itself uses a Kalman filter (KF) that utilizes the output of a maximum likelihood (ML) estimator as measurements for tracking the parameters over time. In our case we use the space-alternating generalized expectation-maximization (SAGE) algorithm introduced by [7] as ML estimator. The model order detection is done at time instants $t$ using the posterior estimation results from a bank of KF using information criteria [6]. 


\section{GEOMETRICAL REPRESENTATION OF MPCS}

In case of a moving $\mathrm{Tx}$ and $\mathrm{Rx}$ as for $\mathrm{T} 2 \mathrm{~T}$ or $\mathrm{C} 2 \mathrm{C}$ communication as presented in [8], the Doppler can be derived in two different ways. First, using the train velocities $\boldsymbol{\nu}_{T}$ and $\nu_{R}$, the Doppler shift can be expressed

$$
\begin{aligned}
f_{d}\left(\varphi_{T}, \varphi_{R}\right)=( & \left\|\boldsymbol{\nu}_{T}\right\| \cos \left(\varphi_{T}(t)\right)+ \\
& \left.\left\|\boldsymbol{\nu}_{R}\right\| \cos \left(\varphi_{R}(t)\right)\right) \cdot \frac{f_{c}}{c} .
\end{aligned}
$$

AoD is represented by $\varphi_{T}$ and AoA by $\varphi_{R}$; the carrier frequency $f_{c}$ is divided by the speed of light $c$.

Second, the Doppler shift can be described as the derivation of the delay change as shown in (3), with $\mathbf{x}_{S}$ as the scatterer position vector [8]:

$$
f_{d}\left(\mathbf{x}_{S}, t\right)=-\frac{\partial}{\partial t} \tau_{i}\left(\mathbf{x}_{S}, t\right) \cdot f_{c}
$$

As well known, propagation paths with a propagation length of $\tau_{i}(t) \cdot c$ results from a single interaction point at scatterer position $\mathbf{x}_{c}$ are located on an ellipse with their focuses at $\mathrm{Tx}$ and $\mathrm{Rx}$ positions. The ellipse is defined as

$$
a_{\tau_{i}(t)}=\frac{\tau_{i}(t) \cdot c}{2}
$$

and

$$
b_{\tau_{i}(t)}=\sqrt{\left(\frac{\tau_{i}(t) \cdot c}{2}\right)^{2}-\left(\frac{\left\|\mathbf{x}_{T}-\mathbf{x}_{R}\right\|}{2}\right)^{2}}
$$

where $a_{\tau_{i}(t)}$ is the semi-major axis and $b_{\tau_{i}(t)}$ the semi-minor axis [9]. The Tx with the position vector $\mathbf{x}_{T}$ and Rx with it's position vector $\mathbf{x}_{R}$ are in the focuses of the ellipse. To further determine $\mathbf{x}_{S}$, geometrical relations for individual MPCs are considered.

\section{A. Geometrical relations between consecutive snapshots}

In the following, we will discriminate between scatterers that are fixed in their positions and time dependent scatterers.

First, we consider a point scatterer like a tree or a mast of the catenary. The scattering position remains the same over time (i.e. $\mathbf{x}_{S}$ time independent) while the $\mathrm{Tx}$ and $\mathrm{Rx}$ positions change. The resulting phase shift of one path from

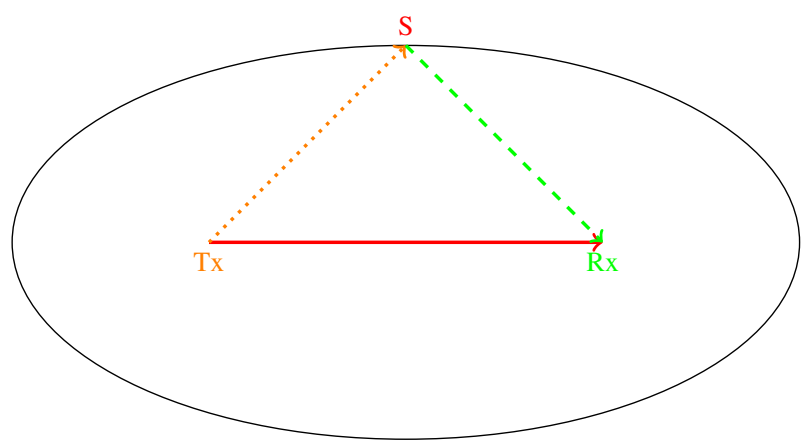

Fig. 1: Geometrical representation of an scatterer, with Tx and $\mathrm{Rx}$ in the focuses of the ellipse. one snapshot to the next snapshot results in a Doppler shift. If we draw ellipses related to the delay of one MPC for each snapshot, the ellipses will intersect in the position of the point scatterer $\mathbf{x}_{S}$ which is static.

Second, for a moving scatterer position like on a flat wall of a house or noise barrier, the triangular relation between $\mathrm{Tx}$, $\mathrm{Rx}$ and the scatterer remains similar over time. If both trains drive with the same speed in the same direction, the Doppler will be close to 0 for this kind of MPC.

\section{B. Geo-information from maps and pictures}

An easy way to identify scatterer points is to use geoinformation from e.g. Google-maps or from videos or pictures. For example, the possible scatterer points can be plotted on top of a map and correlated to objects on the map. The maps can be supplemented with pictures obtained from the measurement campaign.

\section{T2T MEASUREMENT CAMPAIGN}

In April 2016 Trenitalia [10] and the German Aerospace Center (DLR) performed the world wide first T2T C-Band channel sounding measurement campaign with two HSTs. Within four nights we measured the wireless propagation in various environments for different maneuvers on the high speed railway (HSR) between Naples and Rome. The main measurement equipment was the DLR RUSK channel sounder. The channel sounder was set up at a center frequency $f_{c}=$ $5.2 \mathrm{GHz}$ with a bandwidth of $120 \mathrm{MHz}$.

In the third night, T2T propagation with omni-directional antennas was measured. Overtaking and crossing maneuvers in different environments like sub-urban, rural and tunnels were performed. One data set with an overtaking maneuver in a rural environment is used for the presented results in the later sections. In case of the overtaking maneuvers, the Tx train was driving with a speed of $50 \mathrm{~km} / \mathrm{h}$ and the Rx train with a speed varying between $10 \mathrm{~km} / \mathrm{h}$ to $70 \mathrm{~km} / \mathrm{h}$. Further information can be found in [4].

\section{RESUlts}

The following results are based on the measurements from the third night with omni-directional antennas. Two blocks of an overtaking maneuver, with 1200 snapshots each, are picked out and presented. The whole maneuver took place in rural environment; the Rx train with a speed $\nu_{R}=10 \mathrm{~km} / \mathrm{h}$ is in front of the Tx train with a speed of $\nu_{T}=50 \mathrm{~km} / \mathrm{h}$. In the first evaluation block the link distance is around $1250 \mathrm{~m}$ and in the second block of around $60 \mathrm{~m}$ as shown in Figure 2 and 3 .

\section{A. Velocity and Distance Estimation}

To measure the time variant CIR, stationarity must be ensured within the measurement of one snapshot [11]. Therefore, the absolute velocity of the Tx train $\boldsymbol{\nu}_{T}$ and the Rx train $\boldsymbol{\nu}_{R}$ as well as the resulting relative velocity $\Delta \nu$ in (6) need to be chosen. In combination with the time period of the transmitted 
TABLE I: Time settings and maximum velocity

\begin{tabular}{|c|c|c|c|c|}
\hline Setting & $t_{r}$ & $t_{p}$ & \multicolumn{2}{|c|}{$\nu_{\max }$} \\
& {$[\mathrm{ms}]$} & {$[\mu \mathrm{s}]$} & {$[\mathrm{m} / \mathrm{s}]$} & {$[\mathrm{km} / \mathrm{h}]$} \\
\hline 1 & 1.024 & 12.8 & 28.15 & 101.3 \\
2 & 2.048 & 25.6 & 14.08 & 50.7 \\
\hline
\end{tabular}

signal $t_{p}$ and the time between two consecutive snapshots $t_{r}$, (7) and (8) should be fulfilled.

$$
\begin{gathered}
\Delta \nu=\max \left(\left|\boldsymbol{\nu}_{T}-\boldsymbol{\nu}_{R}\right|,\left|\boldsymbol{\nu}_{T}+\boldsymbol{\nu}_{R}\right|\right) \\
t_{p} \cdot \Delta \nu<<\lambda \\
t_{r} \cdot \Delta \nu<\frac{\lambda}{2},
\end{gathered}
$$

where $\lambda$ stands for the wavelength of the transmitted signal. For the used carrier frequency $f_{c}=5.2 \mathrm{GHz}, \lambda$ equals to $5.77 \mathrm{~cm}$.

The given values for $t_{p}$ and $t_{r}$ and resulting maximum velocities $\nu_{\max }$ are listed in Table I. As mentioned before, the Tx train was driving at a constant speed of $50 \mathrm{~km} / \mathrm{h}$ The Rx train varied the speed from $10 \mathrm{~km} / \mathrm{h}$ to fall behind, up to $70 \mathrm{~km} / \mathrm{h}$ to overtake the $\mathrm{Tx}$ train. To obtain time variant speeds, the global navigation satellite system (GNSS) measurements were performed and post processed. With an update rate of $1 \mathrm{~Hz}$ of the GNSS information, every 976 snapshot the velocity and distance can be updated. Due to the large inertial mass of the trains, the change of velocity within $1 \mathrm{~s}$ is rather small.

The Rx velocity information is used to calculate the moved distance of the Rx between two consecutive measurement snapshots. The time $t_{r}$ is stable due to the use of rubidium clocks at Tx and Rx. Therefore, the main limiting factor for the distance estimation accuracy is the velocity estimation of the GNSS receivers.

The distance between $\mathrm{Tx}$ and $\mathrm{Rx}$ can be derived in two ways. First, the distance is calculated by the GNSS positions and, therefore, limited in accuracy. Second, the distance is estimated by the absolute delay of the line of sight (LOS) of each CIR. It is only possible when the LOS path can be accurately tracked by the KEST algorithm.

\section{B. Path Detection and Tracking}

The results of the KEST algorithm for the far and short distance link are shown in Figure 5 and Figure 7 respectively. In comparison, the CIRs of both measurements are plotted in Figure 4 and Figure 6. In all four figures the delay $\tau_{i}$ is plotted over the moved distance of the Rx. The color of each point indicates the power in $\mathrm{dB}$.

The measurement bandwidth is limited to $120 \mathrm{MHz}$. The signal spreading in delay of the LOS path, visible in Figure 4 and Figure 6, results from the rectangular transmitted signal in frequency domain, i.e. a sinc function in time domain. In Figure 4 all MPCs follow clear trends and can be seen easily.

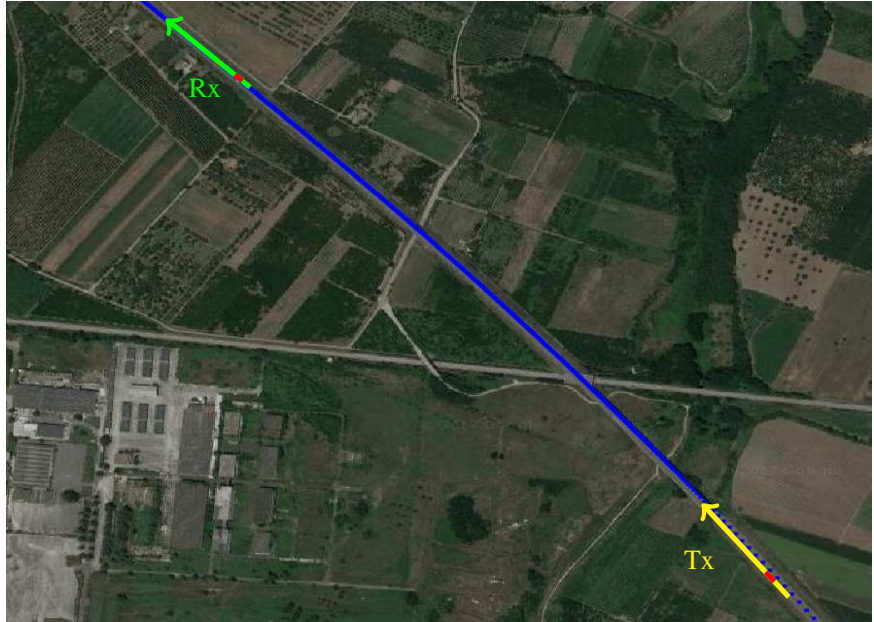

Fig. 2: Track map for far distance scenario. The green arrow indicates the Rx train, the yellow arrow the Tx train and their directions. The blue line/dots represent GNSS measurements, the red lines indicate the antenna position on the trains. Image by Google 2017, Image Landsat/Copernicus (2016).

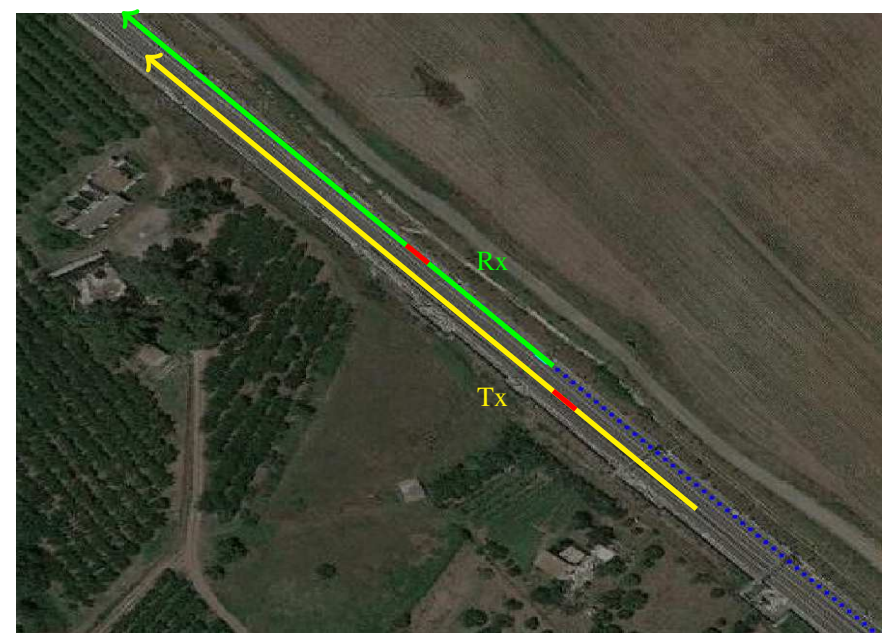

Fig. 3: Track map for short distance scenario. The green arrow indicates the Rx train, the yellow arrow the Tx train and their directions. The blue dots represent GNSS measurements, the red lines indicate the antenna position on the trains. Image by Google 2017, Image Landsat/Copernicus (2016).

In comparison, for the short range measurements the MPCs can not be clearly visually extracted in Figure 6.

The four MPCs displayed in Figure 5 will be described in more detail in the following. The LOS path is continuously tracked starting with a delay of $4.27 \mu$ s which is related to the link distance of $1.28 \mathrm{~km}$. Fading of around $15 \mathrm{~dB}$ can be observed on the LOS path amplitude. MPCs close to the LOS path with additional delay of 5 to $15 \mathrm{~ns}$ might be caused by obstacles of the train structure. Two further MPCs were tracked with similar delay change as the LOS path with an additional offset of $0.26 \mu \mathrm{s}$ and $0.55 \mu \mathrm{s}$. Those MPCs might be caused by the noise barriers located parallel to the track. 


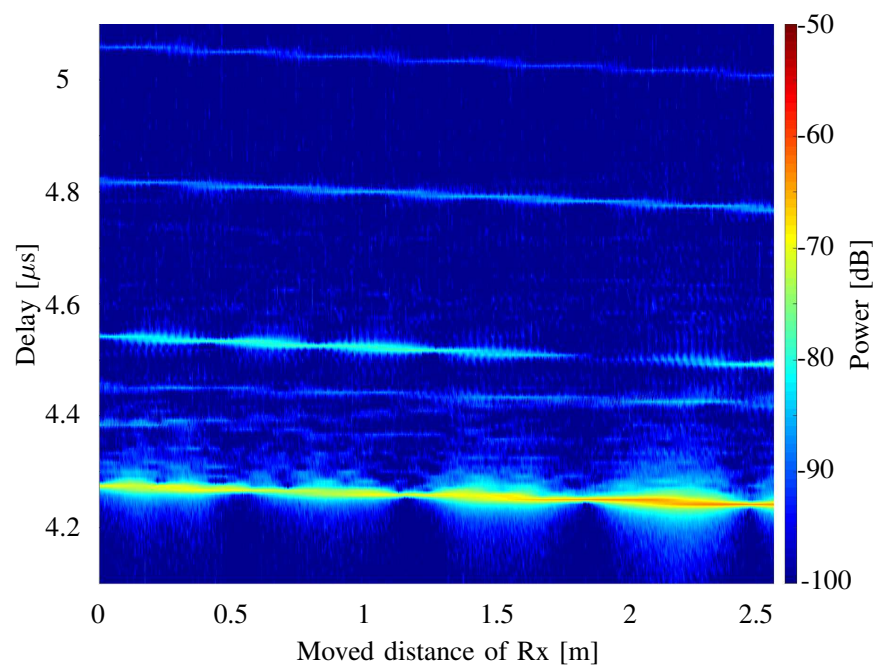

Fig. 4: Raw CIR of the far distance link.

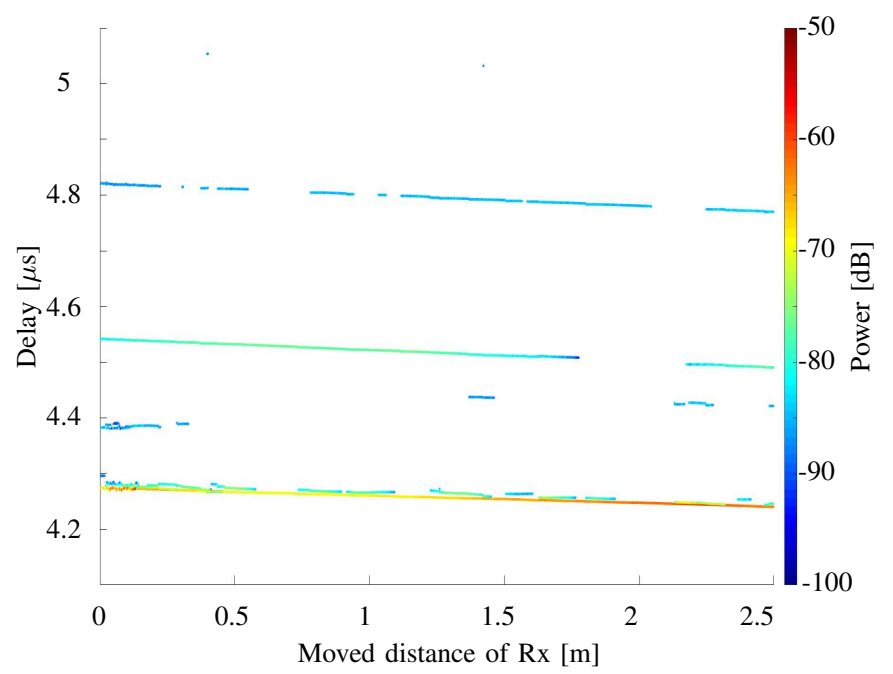

Fig. 5: KEST evaluation of the far distance link.

The second evaluation presented in Figure 7 shows four dominant paths. The LOS path is tracked well with a delay of $0.22 \mu$ s, i.e. a link distance of $66.1 \mathrm{~m}$ at the start. The power varies over a range of $25 \mathrm{~dB}$. Two MPCs are tracked with a similar delay slope being parallel to the LOS starting at $0.37 \mu \mathrm{s}$ and $0.4 \mu \mathrm{s}$. One MPC is starting at $0.37 \mu \mathrm{s}$ and ends at $0.43 \mu \mathrm{s}$ while the delay increases over the moved distance.

\section{CONCLUSION}

In this contribution we presented first results of the postprocessing of T2T channel sounding measurements in HSR environments. The KEST algorithm was used to estimate and track the channel parameters of MPCs over time. The results for two link distances of around $60 \mathrm{~m}$ and $1.2 \mathrm{~km}$ in a rural environment were shown. We could prove that for both measurements the KEST algorithm is estimating the measured CIR and is tracking the MPCs over several meters.

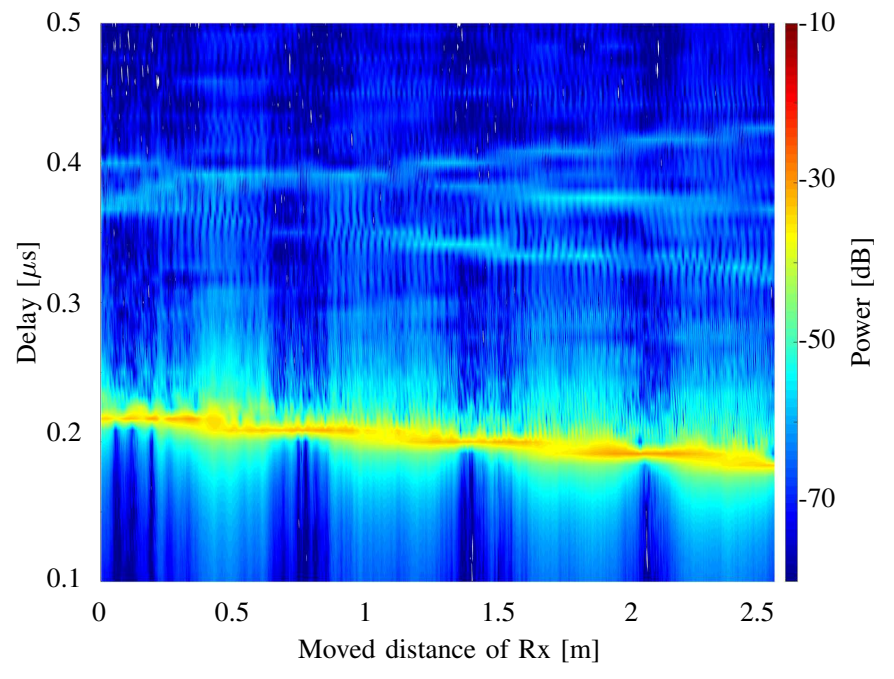

Fig. 6: Raw CIR of the short distance link.

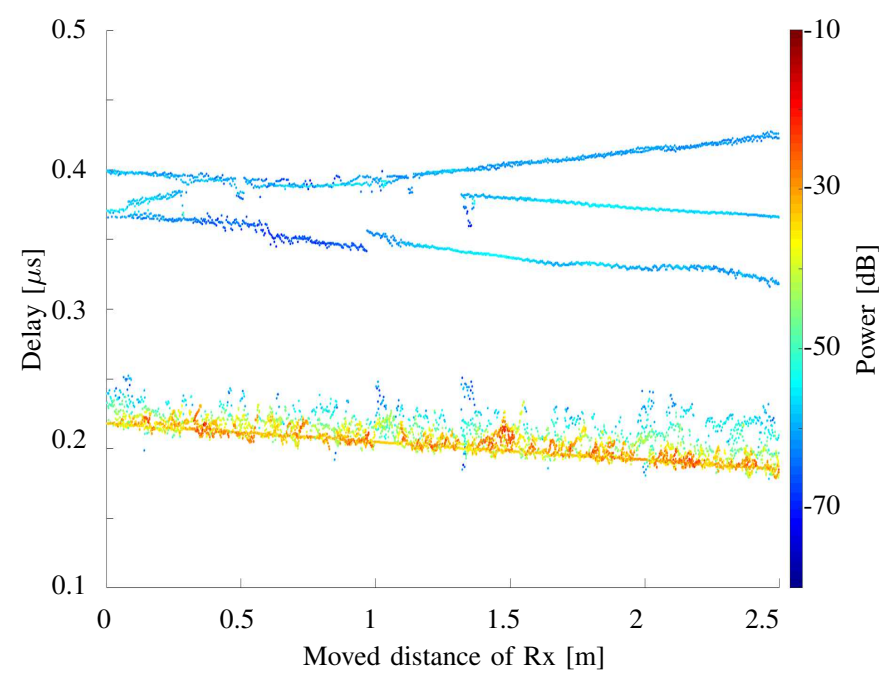

Fig. 7: KEST evaluation of the short distance link.

Due to the SISO measurement setup and the movement of Tx and Rx, we could only estimate the complex amplitude and the delay with KEST. Therefore, the geometrical representation of MPCs using point scatterers and possibilities to identify scattering positions without angular information were presented.

\section{ACKNOWLEDGMENT}

The authors are thankful for the support of the European Commission through the Roll2Rail project [12], one of the lighthouse projects of Shift2Rail [13] within the Horizon 2020 program. The Roll2Rail project has received funding from the European Unions Horizon 2020 research and innovation program under Grant Agreement no. 636032. 


\section{REFERENCES}

[1] P. Unterhuber et al., "A Survey of Channel Measurements and Models for Current and Future Railway Communication Systems," Mobile Information Systems, vol. 2016, pp. 1-14, June 2016. [Online]. Available: http://dx.doi.org/10.1155/2016/7308604

[2] J. Karedal, F. Tufvesson, N. Czink, A. Paier, C. Dumard, T. Zemen, C. F. Mecklenbrauker, and A. F. Molisch, "A Geometry-Based Stochastic MIMO Model for Vehicle-to-Vehicle Communications," IEEE Transactions on Wireless Communications, vol. 8, no. 7, pp. 3646-3657, July 2009.

[3] W. Wang and T. Jost, "A Low-Cost Platform for Time-Variant Wireless Channel Measurements With Application to Positioning," IEEE Transactions on Instrumentation and Measurement, vol. 61, no. 6, pp. 15971604, June 2012.

[4] P. Unterhuber et al., "Wide Band Propagation in Train-to-Train Scenarios - Measurement Campaign and First Results," in 2017 11th European Conference on Antennas and Propagation (EuCAP), March 2017, pp. 3356-3360.

[5] A. F. Molisch, "Ultra-Wide-Band Propagation Channels," Proceedings of the IEEE, vol. 97, no. 2, pp. 353-371, Feb 2009.

[6] T. Jost, W. Wang, U. C. Fiebig, and F. Perez-Fontan, "Detection and Tracking of Mobile Propagation Channel Paths," IEEE Transactions on Antennas and Propagation, vol. 60, no. 10, pp. 4875-4883, Oct 2012.

[7] B. H. Fleury, M. Tschudin, R. Heddergott, D. Dahlhaus, and K. I. Pedersen, "Channel Parameter Estimation in Mobile Radio Environments Using the SAGE Algorithm," IEEE Journal on Selected Areas in Communications, vol. 17, no. 3, pp. 434-450, Mar 1999.

[8] M. Walter, D. Shutin, and U. C. Fiebig, "Delay-Dependent Doppler Probability Density Functions for Vehicle-to-Vehicle Scatter Channels," IEEE Transactions on Antennas and Propagation, vol. 62, no. 4, pp. 2238-2249, April 2014.

[9] I. Bronshtein, K. Semendyayev, G. Musiol, and H. Mhlig, Handbook of Mathematics, 5th ed. Springer-Verlag Berlin Heidelberg, 2007.

[10] Trenitalia, "ETR 500," 2016. [Online]. Available: http://www.trenitalia. com/

[11] P. Bello, "Characterization of Randomly Time-Variant Linear Channels," IEEE Transactions on Communications Systems, vol. 11, no. 4, pp. 360393, December 1963.

[12] Roll2Rrail, 2015. [Online]. Available: http://www.roll2rail.eu/

[13] Shift2Rail, 2015. [Online]. Available: http://www.shift2rail.org 